\title{
Téoros
}

Revue de recherche en tourisme

\section{Le circuit des fleurs}

\section{Normand Cazelais}

Volume 18, numéro 1, printemps 1999

Les jardins du tourisme

URI : https://id.erudit.org/iderudit/1072303ar

DOI : https://doi.org/10.7202/1072303ar

Aller au sommaire du numéro

\section{Éditeur(s)}

Université du Québec à Montréal

\section{ISSN}

0712-8657 (imprimé)

1923-2705 (numérique)

Découvrir la revue

\section{Citer cet article}

Cazelais, N. (1999). Le circuit des fleurs. Téoros, 18(1), 60-63. https://doi.org/10.7202/1072303ar d'utilisation que vous pouvez consulter en ligne.

https://apropos.erudit.org/fr/usagers/politique-dutilisation/ 


\section{Le CIRCUIT DES fLeURS}

\section{Normand Cazelais}

Depuis la plus lointaine Antiquité, les hommes ont accordé une grande importance aux fleurs et aux jardins. De l'Égypte des pharaons aux jardins suspendus de Nabuchodonosor en Mésopotamie, du Japon à l'empire des Aztéques, de la Grèce classique à la Rome glorieuse, ils ont tenu une place centrale dans la vie quotidienne. Réponse au besoin d'ameliorer le décor de tous les jours? Aspiration viscérale à la beauté ? Volonté de projeter une image favorable chez les voisins et les visiteurs, de faire étalage de richesse ou de puissance? Si les paysages naturels ont toujours attiré les voyageurs, l'insertion de jardins et de lieux de fleurs au sein de circuits ou de stratégies de promotion touristique est un phénomène assez récent au Québec.

II n'en demeure pas moins qu'on ne compte plus aujourd'hui, dans les diverses régions du Québec, que ce soit en Montérégie, sur la Côte-du-Sud ou dans les Cantons-de-l'Est, les circuits qui surgissent, alimentés par le fil d'Ariane des jardins et des fleurs. Il est toutefois difficile de se faire une juste idée de l'ampleur du phénomène et de sa contribution au produit touristique québécois en général et dans ses composantes locales ou régionales: le sujet est fort peu documenté, en raison de l'absence quasi totale de données statistiques pertinentes et d'études ou d'enquếtes s'y intéressant. Pour mieux le cerner, il faut donc se tourner vers les acteurs eux-mềmes.

\section{FLEURIR LE QUÉBEC}

En 1958, on lançait le concours Villes. villages et campagnes fleuris du Québec. En 1996, 355 municipalites, soit le quart des municipalités de la province, s'y étaient inscrites. L'année suivante, à l'aube de sa dix-neuvième édition, le ministère de l'Agriculture, des Pêcheries et de l'Alimentation du Québec (MAPAQ) en dressait le bilan suivant :

\begin{abstract}
Au cours des années, ce concours a permis aux municipalités de toutes les régions du Québec de mettre en valeur les efforts qu'elles déploient pour améliorer, grâce à l'embellissement horticole, la qualite de vie de leur population et (pour) créer un environnement sain et agréable. Il constitue également un attrait important de l'industrie touristique régionale.
\end{abstract}

Au printemps 1999, le concours a pris une nouvelle appellation, Fleurir le Québec, et s'est doté d'un nouveau visage tout en renforçant son apport touristique. En partenariat avec le MAPAQ, l'Association des jardins du Québec (AJQ) annonçait que, dans les années à venir, quatre de ses membres allaient à tour de rôle organiser les Fêter régionales où l'on remettrait les prix aux gagnants régionaux et révélerait l'identité des trois municipalités gagnantes au plan national. Lors des célébrations, précisait-on, * les participants seront invités à découvrir, par des activités spéciales (visites guidées, rallye-nature, expositions, etc.), les richesses du jardin-hôte ${ }^{2}$ ». Les jardins retenus à cette fin étaient alors le
Domaine Joly-De Lotbinière (2I août), les Jardins de Métis (29 août), le Pare MarieVictorin (11 septembre) et le Centre de la Nature de Laval (12 septembre).

Le MAPAQ a conservé la responsabilité des Prix nationaux et l'organisation du Gala subséquent. Le document de présentation du ministère ${ }^{3}$ précisait en outre:

Les noms des * municipalités fleuries (i.e. gagnantes des prix nationaux pour chacune des sept catégories) seront dévoilés lors du gala (et) diffusés par le biais de différents outils promotionnels touristiques invitant la population à découvrir les belles municipalités du Québec.

Pour sa part, 1'AJQ rappelait que ses membres ont accueilli en 1998 plus de trois millions et demi de visiteurs, tant québécois qu'étrangers, qui ont laissé des retombées économiques de plusieurs millions de dollars en région ${ }^{4}$.

Le 26 mars 1999 Rémy Trudel, ministre responsable du MAPAQ, invitait les dirigeants municipaux « à soutenir le développement touristique et la capacité d'attraction de leur municipalité en encourageant leur mise en valeur par la beauté et les fleurs *. II annonçait aussi, à cette occasion, le lancement, en collaboration avec Tourisme Québec, d'une brochure touristique «nationale, destinée à faire reconnaître les plus belles municipalitếs fleuries dans chacune des régions $*$ et leur inscription dans les guides annuels des Associations touristiques régionales.

Fleurs et jardins sont done devenus des attractions touristiques reconnues et 
peut-on présumer, rentables. Voyons quelques exemples.

\section{L'EFFLEURE-PRINTEMPS}

A la fin de mars 1999, le promoteur François-Guy Laroque a dô annuler, « faute de local appropriés ", l'EffleurePrintemps, evénement qui attire chaque année près de 25000 visiteurs à Laval, surnommée la capitale de l'horticulture au Québec, grâce à plus de 500 entreprises ceuvrant dans ce secteur et à une production florale en serres représentant près du tiers du total québécois. Cette neuvieme édition devait avoir lieu du 22 au 25 avril. Le président de 1'Office de tourisme de Laval, John d'Ambrosio, a déploré la situation :

D'ici l'an prochain, il est imperatif qu'une salle d'exposition de calibre international soit construite sur le territoine de Laval. Cette salle devrait pouvoir accueillir, tout au long de l'anmée, diverses expositions ou manifestations à caractè re horlicole, floral et agricole aux retombees touristiques et economiques indéniables".

\section{EAST HEREFORD}

Village de $71 \mathrm{~km}^{2}$ des arrières de Coaticook tout près du New Hampshire, East Hereford ( 325 habitants) a remporté le titre de Campagne fleurie du Québec en 1983. 1989 et 1992.

East Hereford, écrit Lucie RoyAlain ${ }^{7}$ secrétaire-trésorière de la municipalité, est choye par whe nature généreuse, une population qui a un bon sentiment d'appartenance, du bénevolat de qualité [...] Notre fierté se reflète dans nos anénagements paysagers. Notre municipalite est avant-gardiste en embellissement et en environnement.

Le virage vert y fut entrepris dès 1982 avec la mise sur pied d'un comitế d'embellissement qui a crét, en 1991, la fondation Bel Environ - première du genre en milieu rural — vouée à l'embellissement à la conservation et à la protection de l'environnement. Ses réalisations sont multiples: compostage domestique et récupération d'arbres de Noël, collecte sélective de porte à porte, récupération de cassettes de machines à écrire et d'imprimantes, de chaussures et de vêtements usagés, d'emballages de plastique, de produits toxiques, etc.

Mais la reconnaissance touristique ne fut pas immédiate; Lucie Roy-Alain ${ }^{5}$ écrit encore

Malgré une participation assidue à Villes, villages et campagnes fleuris du Québec, plusieurs annees se sont écoulées avant qu'East Hereford ne soit reconnu. En 1989, lorsque nous avons gagné un premier prix provincial, aucune mention $n$ 'avait été faite dans les journaux, de nous ou de tous les autres gagnants. Ce sujet passait presque inapercu l... J Une anecdore qui en dit fort long sur ce que valaient alors tous ces prix I Nous souhaitions faine partie du Guide towristique des Cantons-de-l'Est ; nous avons appelé le bureau responsable et demande ce qu il fallait faire pour être whe destination identifiee. Presque insultes, on nous repond que les gens ne se déplaceron certainement pas pour aller voir des flewrs. [...]

En 1992, ce fut wn autre premier prix provincial et, l'année suivante, une selection pour représenter le Canada, avec Saint-Bruno-deMontarville, à l'Entente florale internationale. La médiatisation ecrite et parlée de ce dernier buenement nous a propulsés bien au-delà de ce que nous nous attendions $[. .$. Et les touristes, individuellement d'abord, ont commencé à s'amener à East Mereford pour visiter les jardins fleuris. Des visites de groupe sont maintenant possibles avec visites de jardins et de plantations d'arbres de Noel. Les cincuits Frontienes (velo de montagne et de campagne) attirent we chentele intéressante.

Tous les efforts deployés en embellissement, environnement, tourisme vert et vie communautaire sont devenus un réflexe naturel. Des articles de revues et de quotidiens et plusieurs reportages televisés complètent bien nos effors, nous amenant leur lot de louristes à la recherche d'une nature abondante. Dans un sens, tout est devenu plus facile.
Mais nous sommes également victimes de notre succes. Les municipalités voisines de Saint-Venant-dePaquette, avec son église-musée et I'ouverture du Sentier poétique, et de Saint-Malo, avec satour d'observation La Montagnaise (on parle maintenant de la route des TroisVilages), ajoutent d'autres visiteurs en région et nous devrons, dans un avenir rapproche, penser à une structure d'accueil organisé pour bien renseigner et diriger tout ce beau monde. [...]

Un tel témoignage parle par lui-même et se passe de commentaires.

\section{LE TEMPS DES LILAS}

La réputation touristique de Charlevoix n'est plus à faire, mais tout n'y est pas exploré. La plus técente initiative de Capal-I'Aigle en atteste.

Situé à quatre kilomètres à l'est de La Malbaie et doté d'un panorama remarquable, ce village de 750 habitants attire les touristes depuis le XIX ${ }^{a}$ siecle alors que des estivants anglophones ont commencé à s'y installer. Petit à petit, leur bâti s'est intégré à celui de la population francophone, rurale pour l'essentiel, y implantant entre autres son * amour des jar$\operatorname{dins}^{9}$ \%. Aujourd'hui, Cap-à-l'Aigle est « pourvu d'acquis horticoles et flotaux dont la renommée excède les limites de la région $w$.

Pour en tirer parti, on a mis sur pied une corporation, nommée Cap-à-l'Aigle/Village des Whas. Chargée de développer un concept global, celui d' $*$ intégrer le lilas à un village pittoresque ayant déjà une notoriété touristique $x$, Le temps des lilas en sera le principal événement chaque année, durant quatre jours en juin. Sa programmation comprendra des visites guidées, la présence d'aquarellistes et d'artisans, la présentation de concerts à l'église catholique et à la chapelle protestante St. Peter on the Rock : $\ll$ une rencontre, selon ses organisateurs, entre l'art du

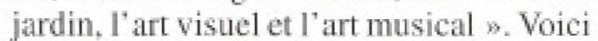
d'ailleurs comment ils expliquent leur approche :

Ce concept répond à une forte tendance mondiale definie par l'évolution en age, en culture et en revenu 
de la plus importante couche démographique du siècle, celle des babyboomers. [... I Si on considere la sensibilisation des Québecois envers I'horticulture et l'envinonnement qui se iraduit aussi dans la popularité de l'événement annuel de Villes et villages fleuris (devenu Fleurir le Quebec), si on retient l'intérêt dejà manifesté par l'Intemational Lilac Society et sa volonté de faire connaitre le produit de Cap-à-l'Aigle à son congrès de l'an 2000. cela démonwe que le développement du concept Cap-à-I'Aigle/Village des lilas est doté d'un potentiel majeur.

Fait à noter, on attend 2500 visiteurs en 1999.

Normand Cazelais est getographe et specialiste en enviromement à Hydro-Québec. Il est Egalemen expert en tourisme. II a publié de nombreux lives, articles et chroniques sur les differentes facentes du récreotourisme national et international.

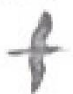

\section{NOTES}

1 (MAPAQ, 1997: 1)

2 (Associations des Jardins du Québec : 1999)

3 (MAPAQ, 1999 ; 3)

4 Pour mieux apprécier l'impact touristique de ces jardins, consulter le tablcau 1 qui présente les membres de l'AJQ et leurs principales caractếristiques.

5 (Office de tourisme de Laval, Laval, 1999 : 1)

6 (Office de tourisme de Laval, Laval, 1999 : 1)

7 Correspondance pertonnelle, août 1998.

8 Correspondance personnelle, le 8 mars 1999.

9 (Corporation Cap-ă-l'Aigle/Village des lilas, $1999: 2$ ) Toutes les autres citations de cette section en sont également tirées.

10 Secrétariat d'Etat au Tourisme, 1999 : Avantpropos.

\section{BIBLIOGRAPHIE}

Ministère de l"Agriculture, des Pêcheries et de I'Alimentation (1997), Points à souligner pour l'édition 1997, Communiqué de presse, Québec. sans date, p. 1.

Associations des Jardins du Québec (1999), Une association pour fleurir le Quebec, Conmuniqué de presse, Québec, 26 marss.

Ministère de l'Agriculture, des Pêcheries et de I'Alimentalion du Québec (1999). Honmage floral - Fleurir le Québec, Québec, mars, p. 3.

Office de tourisme de Laval (1999), Annulation de l'exposition $L$ ' Effeure-Printemps foute d'wne' salle appropriee : l'Office de tourisme de Laval déplore la sifuation, Communiqué de presse, Laval, 24 mars, p. 1

Corporation Cap-ä-l'Aigle/Village des lilas (1999), Le temps des Lilas - La fête des fleurs, Cap-âtl' Aigle, juin, p. 2.

Secrétariat d'État au Tourisme (1999), Balades dans les villes ef villages fleuris, Paris, avantpropos.

\section{AILLEURS}

On sait que Louis XIV fit construire Versailles et aménager ses jardins attenants après avoir vu ce que son intendant Fouquet avait conçu pour ses propres fins à Vauxle-Vicomte. Soupçonné de malversations, Fouquet fut jeté en prison, mais la France gagna un site dont la magnificence inspira les autres cours européennes. Conséquence : en ce continent, fleurs et jardins jalonnent la route des voyageurs depuis des siècles. En Allemagne, en Grande-Bretagne, au bord de la Méditerranée comme de la Baltique. les espaces fleuris sont un intrant reconnu du produit touristique dont les États et les régions font la promotion. Retenons quelques cas.

\section{Aux Pays-Bas}

On identifie ce pays, petit mais très densément peuplé, à ses fleurs, notamment à ses tulipes qui en sont même une image de marque. Deux exemples permettent de relever l'importance qu'on accorde à ce thème dans la stratégie touristique :

- La brochure Welcome Holland 1999-2000. éditée à des millions d'exemplaires, consacre deux pages complètes pour présenter les principaux jardins et parcs du pays, les jardins botaniques, les encans et les circuits, les parades et les autres événements floraux.

- Parmi les voyages de sensibilisation proposés cette année aux chroniqueurs et aux journalistes de voyage, deux sont axés sur les fleurs :

$$
\begin{aligned}
& \text { - Fleurs, fleurs, fleurs (parades et encans). } \\
& \text { - Les jardins de Hollande. }
\end{aligned}
$$

\section{En Itaule}

Longtemps avant son unification, I'Italie était un passage obligé sur l'itinéraire des artistes et des gens de lettres et de pouvoir qui aspiraient à une formation classique. Qu'on pense à Mozart, à Montaigne, à Stendhal. Les jardins floraux étaient, bien sôr, inscrits sur ces circuits. Chaque année, en collaboration avec I'INIT (I'Office de tourisme d' Italie), les directions touristiques régionales italiennes publient des guides couleurs illustrés et des listes, régulièrement mis à jour, des jardins et des pares dans tout le pays.

\section{En France}

Michelle Demessine, Secrétaire d'État au Tourisme et présidente du Comité national pour le fleurissement de la France, ecrit dans le guide 1999 :

Pour la premiere fois celle anné, Balades dans les villes et villages fleuris (répertoriant les 107 communes détentrices du label Quatre fleurs), vous invite à une décowverte originale de nos villes et villages au sommet du concours des Villes et villages fleuris. IPlus foin, la note au lecteur expli. que : / Ces conmunes ont été choisies après une sélection rigourease. pour le soin qu'elles prennent à s'embellir par les fleurs, bien suir, mais aussi par la beauté de leurs parcs. jardins et towes actions permettant d'améliorer le cadre de vie de leurs habitants et d'offrir un accueil souriant à leurs visiteurs 19

Pour chaque commune, le guide mentionne les accès, informe sur l'histoire et décrit les jardins et réalisations végélales remarquables à visiter, les Fêtes vertes, les Fêtes des plantes et les principaux sites touristiques a ne pas manquer sur place et dans les environs. 


\begin{tabular}{|c|c|c|c|}
\hline \multicolumn{4}{|c|}{ TABLEAU 1} \\
\hline Nom & LOCALISATION & Cakacteristidues & $\begin{array}{l}\text { Périodes o" ouverture/ } \\
\text { Tahification }\end{array}$ \\
\hline Arboretum Morgan & Sainte-Anne-de-Bellevue & Arbres cultivess, plantations et forêts naturelles & Tous les jours ; entrée libre \\
\hline Centre de la Nature & Laval & Ráhabilitation d'une carrièré & Tous les jours; ; entrée libre \\
\hline Domaine Cataraqui & Sillery & $\begin{array}{l}\text { Maison-jardin, résidence du } \\
\text { Gouvernement du Québec }\end{array}$ & $\begin{array}{l}\text { Tous les jours, sauf le lundi; } \\
\text { frais d'entrée }\end{array}$ \\
\hline Domaine des Fleurs & Saint-Pacôme & $\begin{array}{l}\text { Jardins naturels sur un million de } \mathrm{pi}^{2} \text {; sentiers } \\
\text { aménagés sur } 1,5 \mathrm{~km} \text {; centre d'histoire de la } \\
\text { foresterie dans le Témiscouata }\end{array}$ & $\begin{array}{l}\text { Tous les jours, à compter de } \\
\text { la Saint-Jean-Baptiste; frais } \\
\text { d'entrée }\end{array}$ \\
\hline Domaine Joly-De Lotbinière & Sainte-Croix-de-Lotbinière & $\begin{array}{l}\text { Parc -jardin datant de la fin du XIXe siècle } \\
\text { auprès d"un manoir et de ses dépendances; } \\
\text { accès au fleuve; arbres rares }\end{array}$ & $\begin{array}{l}\text { De la mi-mai à la mi-octobre; } \\
\text { frais d'entrée }\end{array}$ \\
\hline Domaine Mackenzie-King & $\begin{array}{l}\text { Parc de la Gatineau, } \\
\text { Aylmer/Chelsea }\end{array}$ & $\begin{array}{l}231 \text { ha ; résidence d'été, pendant } 50 \text { ans }_{t} \\
\text { du } 10^{*} \text { premier ministre du Canada }\end{array}$ & $\begin{array}{l}\text { Tous les jours, de la mi-juin à } \\
\text { la mi-octobre; frais d'entrée }\end{array}$ \\
\hline Domaine Maizerets & Québec & Sur un site historique; depuis 1705 & Tous les jours; entrée libre \\
\hline Grands Jardins de Normandin & Normandin & $\begin{array}{l}55 \text { ha; périple à travers les siècles sur } \\
\text { l'w art des jardins } n\end{array}$ & $\begin{array}{l}\text { De la mi-juin ả la fin } \\
\text { septembre; frais d'entrée }\end{array}$ \\
\hline Jardin botanique de Montréal & Montréal & $\begin{array}{l}\text { Fondé en } 1931 \text { par le frère Marie-Victorin : } \\
10 \text { serres d'exposition et une trentaine de } \\
\text { jardins; considéré comme l'un des plus } \\
\text { importants au monde }\end{array}$ & Tous les jours ; frais d'entrée \\
\hline Jardin Daniel A. Séguin & Saint-Hyacinthe & $\begin{array}{l}\text { Aménagé à des fins pédagogiques et } \\
\text { touristiques; thèmes }\end{array}$ & $\begin{array}{l}\text { De juin à septembre; sauf le } \\
\text { lundi ; frais d'entrée }\end{array}$ \\
\hline Jardin Roger Van den Hende & $\begin{array}{l}\text { Pavillon de l'Envirotron, } \\
\text { Université Laval, Sainte-Foy }\end{array}$ & $\begin{array}{l}\text { Fondé en } 1966 \text { par le professeur éponyme; } \\
2000 \text { especes et cultivars regroupés par familles }\end{array}$ & $\begin{array}{l}\text { Du } 1^{\text {si }} \text { mai au } 30 \text { septembre: } \\
\text { entree libre }\end{array}$ \\
\hline Jardins de Métis & Grand-Métis & $\begin{array}{l}20 \text { ha ; créés en } 1926 \text { par Elsie Reford; } \\
\text { plus de } 1000 \text { espèces et variétés }\end{array}$ & $\begin{array}{l}\text { Du début juin à la mi-octobre: } \\
\text { frais d'entrée }\end{array}$ \\
\hline Jardins du Domaine Howard & Sherbrooke & $\begin{array}{l}\text { Ancienne propriété d'un sénateur: } \\
\text { serres municipales }\end{array}$ & $\begin{array}{l}\text { Du } 15 \text { avril au } 15 \text { octobre: } \\
\text { frais d'entrée }\end{array}$ \\
\hline La Roseraie du Témiscouata & Cabano & $\begin{array}{l}\text { Sur le site historique du Fort-Ingall : } \\
1200 \text { rosiers issus de plus de } 250 \text { variétés } \\
\text { et espèces }\end{array}$ & $\begin{array}{l}\text { Tous les jours, de juin à la fin } \\
\text { septembre; frais d'entrée }\end{array}$ \\
\hline Les Jardins à fleur d'eau & Stanbridge-Est & $\begin{array}{l}\text { Boisé de } 6 \text { ha ; plantes aquatiques et de } \\
\text { milieux humides ; sur la Route des vins }\end{array}$ & $\begin{array}{l}\text { De juin à octobre: } \\
\text { frais d'entrée }\end{array}$ \\
\hline Maison Henry-Stuart & Québec & $\begin{array}{l}\text { Jardin privé aménagé entre } 1918 \text { et } 1987 \\
\text { autour d'un cottage de type colonial (1849) }\end{array}$ & Tous les jours ; frais d'entrée \\
\hline Parc des Champs-de-bataille & Québec & $\begin{array}{l}107 \text { ha ; parc historique des \& Plaines } \\
\text { d'Abraham } n \text {; compte plus de } 6000 \\
\text { arbres et le jardin floral Jeanne-d'Arc }\end{array}$ & Tous les jours; entrée libre \\
\hline Parc du Bois-de-Coulonge & Sillery & $\begin{array}{l}24 \text { ha ; parc public sur le lieu de résidence } \\
\text { des lieutenants-gouverneurs de } 1870 \mathrm{a} 1966\end{array}$ & Tous les jours ; entrée libre \\
\hline Parc Marie-Victorin & Kingsey Falls & Aménagé en 1985 ; entretien a écologique z & $\begin{array}{l}\text { De mai à octobre: } \\
\text { frais d'entrée }\end{array}$ \\
\hline Seigneurie des Aulnaies & Saint-Roch-des-Aulnaies & $\begin{array}{l}\text { Domaine du XIX siècle; manoir, moulin à } \\
\text { farine, jardin ornemental, pinéde et guides } \\
\text { en costume d'époque }\end{array}$ & $\begin{array}{l}\text { Tous les jours, de la mi-juin } \\
\text { à la mi-octobre }\end{array}$ \\
\hline Villa Bagatelle & Sillery & $\begin{array}{l}\text { Ancienne résidence privée, gérée par la } \\
\text { tondation éponyme }\end{array}$ & $\begin{array}{l}\text { Tous les jours, sauf le lundi : } \\
\text { frais d'entrée }\end{array}$ \\
\hline
\end{tabular}

